\section{Benefits of mental practice in rehabilitation of patients with stroke}

The efficacy of rehabilitation for patients with hemiparesis following stroke is limited, with a large proportion of individuals unable to use their more affected arm at discharge. Results from several small pilot studies have indicated that mental practice of motor skills could increase the efficacy of motor rehabilitation programs. Now, a phase II, randomized, controlled study has demonstrated the benefit of mental practice in the rehabilitation of patients with chronic stroke.

To compare the efficacy of a rehabilitation program incorporating a mental practice component with that of a control rehabilitation regimen, Page et al. enrolled 32 patients with chronic stroke. In both rehabilitation programs patients received $30 \mathrm{~min}$ traditional therapy sessions, twice a week for 6 weeks during which arm motor skills were physically practiced. In addition, those allocated to the experimental regimen received $30 \mathrm{~min}$ sessions of mental-practice therapy, during which arm motor skills physically practiced in traditional therapy were mentally practiced; those in the control group received 30 min 'sham' interventions involving relaxation exercises. Fine motor function was assessed at study start and 1 week after the completion of therapy using the Action Research Arm Test and the upper extremity section of the Fugl-Meyer Assessment of Motor Recovery After Stroke score. Compared with the control group, those individuals allocated to the experimental program showed a significantly greater increase in movement of the affected arm $(P<0.0001)$ and a significantly greater decrease in arm impairment $(P=0.0001)$. The authors conclude that mental practice is a cost-effective, safe and noninvasive alternative to other, more equipment-intensive, rehabilitation regimens.

Original article Page SJ et al. (2007) Mental practice in chronic stroke: results of a randomized, placebo-controlled trial. Stroke 38: 1293-1297

\section{Can MRI and CSF neurofilament profiles differentiate AD and frontotemporal dementia?}

Alzheimer's disease (AD) and frontotemporal dementia (FTD) are difficult to differentiate clinically because of overlapping symptoms; a conclusive diagnosis can only be made by histopathological analysis at autopsy. A clinical diagnosis is most often made on the basis of extensive history-taking and neuropsychiatric examination. Two recently published studies have attempted to identify surrogate markers for the two disorders, thereby aiding the differential diagnosis of $A D$ and FTD.

Du et al. investigated whether regional maps of the brain's cortical surface, reconstructed using MRI enhanced by automated tools, would reveal characteristic patterns of cortical thinning that could be used to differentiate $A D$ and FTD. They compared the cortical thicknesses of the brains of 22 patients with $A D$, 19 patients with FTD and 22 cognitively normal controls. AD was associated with thinning in the bilateral frontal, parietal, temporal and occipital lobes, whereas the pattern of thinning in FTD mainly involved the frontotemporal lobe and to some extent the posterior cingulated cortex. The results suggested that there was a relationship between cortical thickness and severity of cognitive impairment in AD, but no such association was found in FTD. The investigators concluded that measurement of cortical thinning was approximately as accurate as measurement of cortical volume loss for differentiation of $A D, F T D$ and normal aging. Mapping patterns of cortical thinning increases the clinical usefulness of classification based on neuropsychological scores alone, so cortical thickness measured by MRI could be a useful surrogate marker for $A D$ and FTD.

Recent studies have indicated that neurofilaments are involved in the pathophysiology of FTD. Pijnenburg et al. consequently hypothesized that cerebrospinal fluid (CSF) neurofilaments might have more diagnostic relevance than the biomarkers currently used to differentiate patients with FTD or early onset AD (EAD) from healthy individuals. Neurofilament levels were measured in 17 patients with FTD, 20 patients with EAD and 25 cognitively normal controls. The investigators found that CSF neurofilament profiles were highly variable in FTD, and could not be used to differentiate patients with FTD from those with EAD or from controls. They did find, however, that a subgroup of patients with FTD expressed very high levels of two different neurofilaments, $\mathrm{NfL}$ and $\mathrm{NfH}$, implying a 